\title{
HODGE GROUPS OF CERTAIN SUPERELLIPTIC JACOBIANS II
}

\author{
JIANGWEI XUE
}

\begin{abstract}
We determine the Hodge group of certain simple factor of the Jacobian of the superelliptic curve $y^{q}=f(x)$, assuming that the ground field is a subfield of the complex numbers, $f(x)$ is an degree $n \geq 4$ irreducible polynomial with "large" galois group, and $q$ is a prime power coprime to $n$ and greater than $n$. The case $q<n$ was previous treated in a joint work with Yu.G. Zarhin.
\end{abstract}

\section{Introduction}

Throughout this paper $\mathbf{C}$ is the field of complex numbers, $K \subseteq \mathbf{C}$ is a subfield of $\mathbf{C}$, $f(x) \in K[x]$ a polynomial without multiple roots and of degree $n \geq 4$. Let $p \in \mathbf{N}$ be a prime that does not divide $n$ and $q=p^{r} \in \mathbf{N}$ an integral power of $p$. We write $C_{f, q}$ for the superelliptic $K$-curve $y^{q}=f(x)$, and $J\left(C_{f, q}\right)$ for the Jacobian of $C_{f, q}$. By definition, $C_{f, q}$ is the smooth projective model of the affine curve $y^{q}=f(x)$. The Jacobian $J\left(C_{f, q}\right)$ is an abelian variety over $K$ of dimension

$$
\operatorname{dim} J\left(C_{f, q}\right)=g\left(C_{f, q}\right)=\frac{(n-1)(q-1)}{2} .
$$

If $q>p$, the map

$$
C_{f, q} \rightarrow C_{f, q / p}, \quad(x, y) \mapsto\left(x, y^{p}\right)
$$

induces by Albanese functoriality a surjective $K$-map between the Jacobians $J\left(C_{f, q}\right) \rightarrow$ $J\left(C_{f, q / p}\right)$. We write $J^{(f, q)}$ for the identity component of the kernel. If $q=p$, we set $J^{(f, p)}=J\left(C_{f, p}\right)$. It follows easily that $J^{(f, q)}$ is an abelian variety over $K$ of dimension $(n-1) \varphi(q) / 2$, where $\varphi$ denotes the Euler $\varphi$-function. Moreover, $J\left(C_{f, q}\right)$ is $K$-isogenous to the product $\prod_{i=1}^{r} J^{\left(f, p^{i}\right)}$ (see [15]).

Since $K \subseteq \mathbf{C}$, we may view $J^{(f, q)}$ as a complex abelian variety. We refer to [5; 10, Sections 6.6.1 and 6.6.2] for the definition and basic properties of the Hodge group (aka special Mumford-Tate group). In [9], assuming that $n>q$ and some other conditions on $n, q$ and $f(x)$, the authors showed that the (reductive $\mathbf{Q}$-algebraic connected) Hodge group of $J^{(f, q)}$ coincides with the largest Q-algebraic subgroup of $\operatorname{GL}\left(H^{1}\left(J^{(f, q)}, \mathbf{Q}\right)\right)$ that's "cut out" by the induced polarization from the canonical principal polarization of $J\left(C_{f, q}\right)$ and the endomorphism ring of $J^{(f, q)}$. Notice that when $q=2$ (i.e., in the hyperelliptic case) this group was completely determined in [12] (when $f(x)$ has "large" Galois group). In this paper, we study some additional properties of $J^{(f, q)}$ which will allow us to extend the result to the case $n<q$ as well. This case is necessary in order to treat the infinite towers of superelliptic jacobians, which, in turn, are useful for the study of the ranks of Mordell-Weil groups in infinite towers of function fields (see [6]).

\footnotetext{
Received by the editors January 11, 2011.
} 
To state our main result, we make explicit the endomorphism ring and the polarization mentioned above. Let $X$ be an abelian variety over $\bar{K}$. We write $\operatorname{End}(X)$ for the ring of all its $\bar{K}$-endomorphisms and $\operatorname{End}^{0}(X)$ for the endomorphism algebra $\operatorname{End}(X) \otimes_{\mathbf{Z}} \mathbf{Q}$. In a series of papers [11, 13-15], Yuri Zarhin discussed the structure of $\operatorname{End}^{0}\left(J\left(C_{f, q}\right)\right)$, assuming that $n \geq 5$ and the Galois group $\operatorname{Gal}(f)$ of $f(x)$ over $K$ is, at least, doubly transitive. $\operatorname{Here} \operatorname{Gal}(f) \subseteq \mathbf{S}_{n}$ is viewed as a permutation group on the roots of $f(x)$. It is well known that $f(x)$ is irreducible over $K$ if and only if $\mathrm{Gal}(f)$ acts transitively on the roots. For the sake of simplicity let us assume that $K$ contains a primitive $q$ th root of unity $\zeta_{q}$. The curve $C_{f, q}: y^{q}=f(x)$ admits the obvious periodic automorphism

$$
\delta_{q}: C_{f, q} \rightarrow C_{f, q}, \quad(x, y) \mapsto\left(x, \zeta_{q} y\right) .
$$

By an abuse of notation, we also write $\delta_{q}$ for the induced automorphism of $J\left(C_{f, q}\right)$. The subvariety $J^{(f, q)}$ is $\delta_{q}$-invariant and we have an embedding

$$
\mathbf{Z}\left[\zeta_{q}\right] \hookrightarrow \operatorname{End}\left(J^{(f, q)}\right), \quad \zeta_{q} \mapsto \delta_{q}
$$

In particular, the $q$ th cyclotomic filed $E:=\mathbf{Q}\left(\zeta_{q}\right)$ is contained in $\operatorname{End}^{0}\left(J^{(f, q)}\right)$. Zarhin showed $[11,15,17]$ that $\operatorname{End}\left(J^{(f, q)}\right)$ is isomorphic to $\mathbf{Z}\left[\zeta_{q}\right]$ if either $\operatorname{Gal}(f)$ coincides with the full symmetric group $\mathbf{S}_{n}, n \geq 4$ and $p \geq 3$, or $\operatorname{Gal}(f)$ coincides with the alternating group $\mathbf{A}_{n}$ (or $\mathbf{S}_{n}$ ), and $n \geq 5$. This result has also been extended to the case $\operatorname{Gal}(f)=\mathbf{S}_{n}$ or $\mathbf{A}_{n}, n \geq 5$ and $p \mid n$ in [7].

The first rational homology group $H_{1}\left(J^{(f, q)}, \mathbf{Q}\right)$ carries a natural structure of $E$ vector space of dimension

$$
\operatorname{dim}_{E} H_{1}\left(J^{(f, q)}, \mathbf{Q}\right)=\frac{\operatorname{dim}_{\mathbf{Q}} H_{1}\left(J^{(f, q)}, \mathbf{Q}\right)}{[E: \mathbf{Q}]}=\frac{2 \operatorname{dim} J^{(f, q)}}{[E: \mathbf{Q}]}=\frac{(n-1) \varphi(q)}{\varphi(q)}=n-1 .
$$

Notice that if $q>2$, then $E$ is a CM field with complex conjugation $e \mapsto \bar{e}$. Let

$$
E^{+}=\left\{e \in \mathbf{Q}\left(\zeta_{q}\right) \mid \bar{e}=e\right\}
$$

be the maximal totally real subfield of $E$ and let

$$
E_{-}=\left\{e \in \mathbf{Q}\left(\zeta_{q}\right) \mid \bar{e}=-e\right\} .
$$

The canonical principal polarization on $J\left(C_{f, q}\right)$ induces a polarization on $J^{(f, q)}$, which gives rise to a nondegenerate $E$-sesquilinear Hermitian form [9]

$$
\phi_{q}: H_{1}\left(J^{(f, q)}, \mathbf{Q}\right) \times H_{1}\left(J^{(f, q)}, \mathbf{Q}\right) \rightarrow E .
$$

We write $U\left(H_{1}\left(J^{(f, q)}, \mathbf{Q}\right), \phi_{q}\right)$ for the unitary group of $\phi_{q}$ of the $\mathbf{Q}\left(\zeta_{q}\right)$-vector space $H_{1}\left(J^{(f, q)}, \mathbf{Q}\right)$, viewed as an $\mathbf{Q}$-algebraic subgroup of $\operatorname{GL}\left(H_{1}\left(J^{(f, q)}, \mathbf{Q}\right)\right)$ (via Weil's restriction of scalars from $E^{+}$to $\left.\mathbf{Q}[5]\right)$. Since the Hodge group respects the polarization and commutes with endomorphisms of $J^{(f, q)}$,

$$
\operatorname{Hdg}\left(J^{(f, q)}\right) \subset U\left(H_{1}\left(J^{(f, q)}, \mathbf{Q}\right), \phi_{q}\right) .
$$

If $\operatorname{End}^{0}\left(J^{(f, q)}\right)=E$, then $U\left(H_{1}\left(J^{(f, q)}, \mathbf{Q}\right), \phi_{q}\right)$ is the largest connected reductive Q-algebraic subgroup of $\operatorname{GL}\left(H_{1}\left(J^{(f, q)}, \mathbf{Q}\right)\right)$ that both respects the polarization and commutes with endomorphisms of $J^{(f, q)}$. 
The following theorem is a natural extension of [9, Theorem 0.1].

Theorem 1.1. Suppose that $n \geq 4$ and $p$ is a prime that does not divide $n$. Let $f(x) \in \mathbf{C}[x]$ be a degree $n$ polynomial without multiple roots. Let $r$ be a positive integer and $q=p^{r}$. Suppose that there exists a subfield $K$ of $\mathbf{C}$ that contains all the coefficients of $f(x)$. Let us assume that $f(x)$ is irreducible over $K$ and the Galois group $\operatorname{Gal}(f)$ of $f(x)$ over $K$ is either $\mathbf{S}_{n}$ or $\mathbf{A}_{n}$. Assume additionally that either $n \geq 5$ or $n=4$ and $\operatorname{Gal}(f)=\mathbf{S}_{4}$.

Suppose that one of the following three conditions holds:

(A) $n=q+1$;

(B) $p$ is odd and $n \not \equiv 1 \bmod q$;

(C) $p=2, n \not \equiv 1 \bmod q$ and $n \not \equiv q-1 \bmod 2 q$.

Then $\operatorname{Hdg}\left(J^{(f, q)}\right)=U\left(H_{1}\left(J^{(f, q)}, \mathbf{Q}\right), \phi_{q}\right)$.

Corollary 1.1. Corollary 0.3, Theorems 4.2 and 4.3 of [9] all hold without the assumption that $n>q$.

Remark 1.1. We assume that $n<q$ throughout the rest of the paper since the case $n>q$ has already been treated in [9]. Note that when $q>n$, if $p$ is odd, condition (B) is automatically satisfied; if $p=2$, condition (C) is satisfied as long as $n \neq q-1$.

Remark 1.2. Both $\operatorname{Hdg}\left(J^{(f, q)}\right)$ and $U\left(H_{1}\left(J^{(f, q)}, \mathbf{Q}\right), \phi_{q}\right)$ are connected Q-algebraic groups. So to prove Theorem 1.1, it suffices to show that

$$
\operatorname{dim} \operatorname{Hdg}\left(J^{(f, q)}\right) \geq \operatorname{dim} U\left(H_{1}\left(J^{(f, q)}, \mathbf{Q}\right), \phi_{q}\right) .
$$

It is known that

$$
\operatorname{dim} U\left(H_{1}\left(J^{(f, q)}, \mathbf{Q}\right), \phi_{q}\right)=\operatorname{dim}_{\mathbf{Q}} E^{+} \cdot\left(\operatorname{dim}_{E} H_{1}\left(J^{(f, q)}, \mathbf{Q}\right)\right)^{2} .
$$

Let hdg be the Q-Lie algebra of $\operatorname{Hdg}\left(J^{(f, q)}\right)$. It is a reductive Q-Lie subalgebra of $\operatorname{End}_{\mathbf{Q}}\left(H_{1}\left(J^{(f, q)}, \mathbf{Q}\right)\right)$, and thus splits into a direct sum

$$
\operatorname{hdg}=\mathfrak{c} \oplus \operatorname{hdg}^{\mathrm{ss}},
$$

of its center $\mathfrak{c}$ and the semisimple part $\mathrm{hdg}^{\mathrm{ss}}=[\mathrm{hdg}, \mathrm{hdg}]$. By Xue and Zarhin [8, Theorem 1.3], if $\operatorname{Gal}(f)=\mathbf{S}_{n}$ and $n \geq 4$, or $\operatorname{Gal}(f)=\mathbf{A}_{n}$ and $n \geq 5$, the center $\mathfrak{c}$ coincides with $E_{-}$. Notice that

$$
\operatorname{dim}_{\mathbf{Q}} E_{-}=\operatorname{dim}_{\mathbf{Q}} E^{+}=[E: \mathbf{Q}] / 2 .
$$

Theorem 1.1 follows if we show that:

$$
\operatorname{dim}_{\mathbf{Q}} \operatorname{hdg}^{\mathrm{ss}} \geq \frac{1}{2}[E: \mathbf{Q}]\left(\left(\operatorname{dim}_{E} H_{1}\left(J^{(f, q)}, \mathbf{Q}\right)\right)^{2}-1\right) .
$$

The paper is organized as follows. In Section 2, we study the Galois actions on certain vector spaces. In Section 3, we recall some facts about the Hodge Lie algebra hdg. The proof of Theorem 1.1 is given at the end of Section 3 except a key arithmetic lemma, which is proven in Section 4. 


\section{Galois actions}

Throughout this section, let $E$ be a field that is a finite Galois extension of $\mathbf{Q}$ with Galois group $G$. Let $V$ be a $E$-vector space of finite dimension. We write $V_{\mathbf{Q}}$ for the underlying $\mathbf{Q}$-vector space of $V$, and $V_{\mathbf{C}}$ for the $\mathbf{C}$-vector space $V \otimes_{\mathbf{Q}} \mathbf{C}=V_{\mathbf{Q}} \otimes_{\mathbf{Q}} \mathbf{C}$. Let $\operatorname{Aut}(\mathbf{C})$ be the group of all automorphisms of $\mathbf{C}$. It act semilinearly on $V_{\mathbf{C}}=$ $V \otimes_{\mathbf{Q}} \mathbf{C}$ through the second factor. More explicitly, $\forall \kappa \in \operatorname{Aut}(\mathbf{C}), v \otimes z \in V \otimes_{\mathbf{Q}} \mathbf{C}$, we define $\kappa(v \otimes z):=v \otimes \kappa(z)$. It follows that $\forall x \in V \otimes_{\mathbf{Q}} \mathbf{C}$ and $c \in \mathbf{C}, \kappa(c x)=\kappa(c) x$. On the other hand, $E$ acts on $V_{\mathbf{C}}=V \otimes_{\mathbf{Q}} \mathbf{C}$ through its first factor. It follows that $V_{\mathbf{C}}$ is a free $E \otimes_{\mathbf{Q}} \mathbf{C}$ module of rank $\operatorname{dim}_{E} V$, and the action of $E=E \otimes 1 \subseteq E \otimes_{\mathbf{Q}} \mathbf{C}$ commutes with that of $\operatorname{Aut}(\mathbf{C})$. In other words,

$$
\kappa((e \otimes 1) x)=(e \otimes 1) \kappa(x), \quad \forall \kappa \in \operatorname{Aut}(\mathbf{C}), e \in E, \text { and } x \in V_{\mathbf{C}} .
$$

Let us fix an embedding $E \hookrightarrow \mathbf{C}$. This allows us to identify each Galois automorphism $\sigma: E \rightarrow E$ with the embedding $\sigma: E \rightarrow E \subset \mathbf{C}$ of $E$ into $\mathbf{C}$. It is well known that

$$
E_{\mathbf{C}}:=\mathrm{E} \otimes_{\mathbf{Q}} \mathbf{C}=\bigoplus_{\sigma \in G} E \otimes_{E, \sigma} \mathbf{C}=\bigoplus_{\sigma \in G} \mathbf{C}_{\sigma}, \text { where } \mathbf{C}_{\sigma}:=E \otimes_{E, \sigma} \mathbf{C}
$$

So every $E_{\mathbf{C}}$ module $W$ splits as a direct sum $W=\oplus_{\sigma \in G} W_{\sigma}$, where

$$
W_{\sigma}:=\mathbf{C}_{\sigma} W=\{w \in W \mid(e \otimes 1) w=\sigma(e) w, \forall e \in E\} .
$$

In particular, $V_{\mathbf{C}}=\oplus_{\sigma \in G} V_{\sigma}$, and each $V_{\sigma}$ is a $\mathbf{C}$-vector space of dimension $\operatorname{dim}_{E} V$. For each $\sigma \in G$, let $P_{\sigma}: V_{\mathbf{C}} \rightarrow V_{\sigma}$ be the $\mathbf{C}$-linear projection map from $V_{\mathbf{C}}$ to the summand $V_{\sigma}$. Similarly, for each pair $\sigma \neq \tau$, we write $P_{\sigma, \tau}=P_{\sigma} \oplus P_{\tau}: V_{\mathbf{C}} \rightarrow V_{\sigma} \oplus V_{\tau}$ for the projection map onto this pair of summands.

We claim that $\operatorname{Aut}(\mathbf{C})$ permutes the set $\left\{V_{\sigma} \mid \sigma \in G\right\}$, and the action factors through the canonical restriction

$$
\operatorname{Aut}(\mathbf{C}) \rightarrow G,\left.\quad \kappa \mapsto \kappa\right|_{E} .
$$

Indeed, for all $\kappa \in \operatorname{Aut}(\mathbf{C}), e \in E$ and $x_{\sigma} \in V_{\sigma}$,

$$
(e \otimes 1) \kappa\left(x_{\sigma}\right)=\kappa\left((e \otimes 1) x_{\sigma}\right)=\kappa\left(\sigma(e) x_{\sigma}\right)=\kappa(\sigma(e)) \kappa\left(x_{\sigma}\right)=\kappa \sigma(e) \kappa\left(x_{\sigma}\right) .
$$

Clearly $\kappa \sigma(e)=\left(\left(\left.\kappa\right|_{E}\right) \sigma\right)(e)$. By an abuse of notation, we write $\kappa$ for the restriction $\left.\kappa\right|_{E}$. So it follows that $\kappa\left(x_{\sigma}\right) \in V_{\kappa \sigma}$, and thus $\kappa\left(V_{\sigma}\right)=V_{\kappa \sigma}$ for all $\kappa \in \operatorname{Aut}(\mathbf{C})$ and $\sigma \in G$.

Let us define an action of $\operatorname{Aut}(\mathbf{C})$ on the set of projection $\mathcal{P}=\left\{P_{\sigma} \mid \sigma \in G\right\}$ by

$$
\kappa_{*} P_{\sigma}:=\kappa \circ P_{\sigma} \circ \kappa^{-1} \text {. }
$$

Then for any element $\sum x_{\sigma} \in \oplus_{\sigma \in G} V_{\sigma}=V_{\mathbf{C}}$ and $P_{\tau} \in \mathcal{P}$,

$$
\left(\kappa_{*} P_{\tau}\right)\left(\sum x_{\sigma}\right)=\kappa \circ P_{\tau}\left(\sum \kappa^{-1}\left(x_{\sigma}\right)\right)=\kappa\left(\kappa^{-1}\left(x_{\kappa \tau}\right)\right)=x_{\kappa \tau},
$$

where all summations runs through $\sigma \in G$, and we used the fact that $\kappa^{-1}\left(x_{\sigma}\right)$ belongs to $V_{\tau}$ if and only if $\sigma=\kappa \tau$. Therefore,

$$
\kappa_{*} P_{\sigma}=P_{\kappa \sigma} .
$$


Clearly $\operatorname{Aut}(\mathbf{C})$ acts transitively on $\mathcal{P}$. Since $P_{\sigma, \tau}=P_{\sigma} \oplus P_{\tau}$, we have similarly an action of $\operatorname{Aut}(\mathbf{C})$ on the set $\mathcal{P} \mathcal{P}:=\left\{P_{\sigma, \tau} \mid(\sigma, \tau) \in G^{2}, \sigma \neq \tau\right\}$ by

$$
\kappa_{*} P_{\sigma, \tau}=\kappa \circ P_{\sigma, \tau} \circ \kappa^{-1}=P_{\kappa \sigma, \kappa \tau} .
$$

The $\operatorname{Aut}(\mathbf{C})$-orbit $O_{\sigma, \tau}$ of each $P_{\sigma, \tau} \in \mathcal{P} \mathcal{P}$ consists of all elements of the form $P_{\kappa \sigma, \kappa \tau}$ with $\kappa \in \operatorname{Aut}(\mathbf{C})$.

Lemma 2.1. Let $W_{\mathbf{Q}} \subseteq V_{\mathbf{Q}}$ be any $\mathbf{Q}$-subspace of $V_{\mathbf{Q}}$, and $W_{\mathbf{C}}:=W_{\mathbf{Q}} \otimes_{\mathbf{Q}} \mathbf{C} \subseteq V_{\mathbf{C}}$ be its complexification.

(i) If there exists $\sigma_{0} \in G$ such that $P_{\sigma_{0}}\left(W_{\mathbf{C}}\right)=V_{\sigma_{0}}$, then $P_{\sigma}\left(W_{\mathbf{C}}\right)=V_{\sigma}$ for all $\sigma \in G$.

(ii) If there exists a pair $\left(\sigma_{0}, \tau_{0}\right) \in G^{2}$ with $\sigma_{0} \neq \tau_{0}$ such that $P_{\sigma_{0}, \tau_{0}}\left(W_{\mathbf{C}}\right)=$ $V_{\sigma_{0}} \oplus V_{\tau_{0}}$, then $P_{\sigma, \tau}\left(W_{\mathbf{C}}\right)=V_{\sigma} \oplus V_{\tau}$ for all $P_{\sigma, \tau} \in O_{\sigma_{0}, \tau_{0}}$.

Proof. Clearly, $W_{\mathbf{C}}$ is $\operatorname{Aut}(\mathbf{C})$-invariant. For each $\sigma \in G$, let us choose $\kappa \in \operatorname{Aut}(\mathbf{C})$ such that $\sigma=\kappa \sigma_{0}$. Then

$$
P_{\sigma}\left(W_{\mathbf{C}}\right)=\left(\kappa_{*} P_{\sigma_{0}}\right)\left(W_{\mathbf{C}}\right)=\kappa \circ P_{\sigma_{0}} \circ \kappa^{-1}\left(W_{\mathbf{C}}\right)=\kappa \circ P_{\sigma_{0}}\left(W_{\mathbf{C}}\right)=\kappa\left(V_{\sigma_{0}}\right)=V_{\sigma} .
$$

This proves part (i). Similarly, suppose that $P_{\sigma_{0}, \tau_{0}}\left(W_{\mathbf{C}}\right)=V_{\sigma_{0}} \oplus V_{\tau_{0}}$. For all $P_{\sigma, \tau} \in$ $O_{\sigma_{0}, \tau_{0}}$, there exists $\kappa \in \operatorname{Aut}(\mathbf{C})$ such that $\sigma=\kappa \sigma_{0}$ and $\tau=\kappa \tau_{0}$. So we have

$$
\begin{aligned}
P_{\sigma, \tau}\left(W_{\mathbf{C}}\right) & =\left(\kappa_{*} P_{\sigma_{0}, \tau_{0}}\right)\left(W_{\mathbf{C}}\right)=\kappa \circ P_{\sigma_{0}, \tau_{0}} \circ \kappa^{-1}\left(W_{\mathbf{C}}\right)=\kappa \circ P_{\sigma_{0}, \tau_{0}}\left(W_{\mathbf{C}}\right) \\
& =\kappa\left(V_{\sigma_{0}} \oplus V_{\tau_{0}}\right)=\kappa\left(V_{\sigma_{0}}\right) \oplus \kappa\left(V_{\tau_{0}}\right)=V_{\sigma} \oplus V_{\tau},
\end{aligned}
$$

and part (ii) follows.

Let $R$ be a commutative ring with unity, and $N$ be a free $R$-module of finite rank. We write $\operatorname{Tr}_{R}: \operatorname{End}_{R}(N) \rightarrow R$ for the trace map, and

$$
\mathfrak{s l}_{R}(N):=\left\{g \in \operatorname{End}_{R}(N) \mid \operatorname{Tr}_{R}(g)=0\right\},
$$

for the $R$-Lie algebra of traceless endomorphisms of $N$. It is well known that

$$
\mathfrak{s l}_{E}(V) \otimes_{\mathbf{Q}} \mathbf{C}=\mathfrak{s l}_{E_{\mathbf{C}}}\left(V_{\mathbf{C}}\right)=\mathfrak{s l}_{E_{\mathbf{C}}}\left(\oplus_{\sigma \in G} V_{\sigma}\right)=\bigoplus_{\sigma \in G} \mathfrak{s l}_{\mathbf{C}}\left(V_{\sigma}\right)
$$

We will denote the projection map $\mathfrak{s l}_{E}(V) \otimes_{\mathbf{Q}} \mathbf{C} \rightarrow \mathfrak{s l}_{\mathbf{C}}\left(V_{\sigma}\right)$ again by $P_{\sigma}$, and similarly for $P_{\sigma, \tau}$. Clearly, each $\mathfrak{s l}_{\mathbf{C}}\left(V_{\sigma}\right)$ has $\mathbf{C}$-dimension $\left(\operatorname{dim}_{E} V\right)^{2}-1$.

For the rest of the section, we assume additionally that $E$ is a CM-field. For any $\sigma \in G$, let $\bar{\sigma}: E \rightarrow E$ be the complex conjugation of $\sigma$. In other words, $\bar{\sigma}$ is the composition $E \stackrel{\sigma}{\rightarrow} E \rightarrow E$, where the second arrow stands for the complex conjugation $\operatorname{map} e \mapsto \bar{e}$.

Lemma 2.2. Let $\mathfrak{k}$ be a semisimple $\mathbf{Q}$-Lie subalgebra of $\mathfrak{s l}_{E}(V)$, and $\mathfrak{k}_{\mathbf{C}}:=\mathfrak{k} \otimes_{\mathbf{Q}} \mathbf{C}$ be its complexification. Suppose that the following two conditions hold:

(I) there exists $\sigma_{0} \in G$ such that $P_{\sigma_{0}}\left(\mathfrak{k}_{\mathbf{C}}\right)=\mathfrak{s l}_{\mathbf{C}}\left(V_{\sigma_{0}}\right)$;

(II) For each pair $(\sigma, \tau) \in G^{2}$ with $\sigma \neq \tau$ and $\sigma \neq \bar{\tau}$, there exists $P_{\sigma_{0}, \tau_{0}} \in O_{\sigma, \tau}$ such that $P_{\sigma_{0}, \tau_{0}}\left(\mathfrak{k}_{\mathbf{C}}\right)=\mathfrak{s l}_{\mathbf{C}}\left(V_{\sigma_{0}}\right) \oplus \mathfrak{s l}_{\mathbf{C}}\left(V_{\tau_{0}}\right)$.

Then

$$
\operatorname{dim}_{\mathbf{Q}} \mathfrak{k} \geq \frac{1}{2}[E: \mathbf{Q}]\left(\left(\operatorname{dim}_{E} V\right)^{2}-1\right)
$$


Proof. Applying Lemma 2.1 with $\mathfrak{k}$ in place of $W$ and $\mathfrak{s l}_{E}(V)$ in place of $V$, we see that

$$
\begin{gathered}
P_{\sigma}\left(\mathfrak{k}_{\mathbf{C}}\right)=\mathfrak{s l}_{\mathbf{C}}\left(V_{\sigma}\right), \quad \forall \sigma \in G \\
P_{\sigma, \tau}\left(\mathfrak{k}_{\mathbf{C}}\right)=\mathfrak{s l}_{\mathbf{C}}\left(V_{\sigma}\right) \oplus \mathfrak{s l}_{\mathbf{C}}\left(V_{\tau}\right), \quad \forall(\sigma, \tau) \in G^{2} \text { with } \sigma \neq \tau \text { and } \sigma \neq \bar{\tau} .
\end{gathered}
$$

Let us fix a CM-type $\Phi$ of $E$. By definition, $\Phi$ is a maximal subset of $G=$ $\operatorname{Hom}(E, \mathbf{C})$ such that no two elements of $\Phi$ are complex conjugate to each other. Clearly, $|\Phi|=[E: \mathbf{Q}] / 2$, and

$$
\operatorname{dim}_{\mathbf{C}}\left(\bigoplus_{\sigma \in \Phi} \mathfrak{s l}_{\mathbf{C}}\left(V_{\sigma}\right)\right)=\frac{1}{2}[E: \mathbf{Q}]\left(\operatorname{dim}_{E}(V)^{2}-1\right)
$$

Let $\mathfrak{k}_{\mathbf{C}}^{\prime}$ be the projection of $\mathfrak{k}_{\mathbf{C}}$ on $\oplus_{\sigma \in \Phi} \mathfrak{s l}_{\mathbf{C}}\left(V_{\sigma}\right)$. It follows that the projection $\mathfrak{k}_{\mathbf{C}}^{\prime} \rightarrow$ $\mathfrak{s l}_{\mathbf{C}}\left(V_{\sigma}\right)$ is surjective for all $\sigma \in \Phi$, and $\mathfrak{k}_{\mathbf{C}}^{\prime}$ also projects surjectively onto $\mathfrak{s l}_{\mathbf{C}}\left(V_{\sigma}\right) \oplus$ $\mathfrak{s l}_{\mathbf{C}}\left(V_{\tau}\right)$ for all distinct pairs $\sigma, \tau \in \Phi$. Therefore, $\mathfrak{k}_{\mathbf{C}}^{\prime}=\oplus_{\sigma \in \Phi} \mathfrak{s l}_{\mathbf{C}}\left(V_{\sigma}\right)$ by the Lemma on pp. 790-791 of [4]. In particular, we obtain

$$
\operatorname{dim}_{\mathbf{Q}} \mathfrak{k}=\operatorname{dim}_{\mathbf{C}} \mathfrak{k}_{\mathbf{C}} \geq \operatorname{dim}_{\mathbf{C}} \mathfrak{k}_{\mathbf{C}}^{\prime}=\frac{1}{2}[E: \mathbf{Q}]\left(\left(\operatorname{dim}_{E} V\right)^{2}-1\right) .
$$

In the next section, we will show that our semisimple part of Hodge Lie algebra $\mathrm{hdg}^{\mathrm{ss}}=[\mathrm{hdg}, \mathrm{hdg}]$ satisfies (I) and (II) of Lemma 2.2 and thus prove our main theorem.

\section{The Hodge Lie algebra}

We keep all notation and assumptions of the previous sections. More specifically, $\zeta_{q}$ is a primitive $q$ th root of unity, $E=\mathbf{Q}\left(\zeta_{q}\right)$ and $G=\operatorname{Gal}(E / \mathbf{Q})=(\mathbf{Z} / q \mathbf{Z})^{*}$, where each $a \in(\mathbf{Z} / q \mathbf{Z})^{*}$ maps $\zeta_{q}$ to $\zeta_{q}^{a}$. To simplify the notation, we write $X$ for the abelian variety $J^{(f, q)}$, and $V$ for its first rational homology group $H_{1}(X, \mathbf{Q})$. In addition, we assume that $\operatorname{End}^{0}(X)=E$.

Recall that $E_{\mathbf{C}}=E \otimes_{\mathbf{Q}} \mathbf{C}$. Let $\operatorname{Lie}(X)$ be the complex tangent space to the origin of $X$. By functoriality, $E=\operatorname{End}^{0}(X)$ acts on $\operatorname{Lie}(X)$ and provides $\operatorname{Lie}(X)$ with a natural structure of $E_{\mathbf{C}}$-module. Therefore, $\operatorname{Lie}(X)$ splits into a direct sum

$$
\operatorname{Lie}(X)=\bigoplus_{a \in G} \operatorname{Lie}(X)_{a}
$$

where $\operatorname{Lie}(X)_{a}:=\left\{x \in \operatorname{Lie}(X) \mid\left(\zeta_{q} \otimes 1\right) x=\zeta_{q}^{a} x\right\}$. Let us put $n_{a}=\operatorname{dim}_{\mathbf{C}} \operatorname{Lie}(X)_{a}$. It is known that $n_{a}=[n a / q]$ (see $[15,16]$ ), where $[x]$ is the maximal integer that is less or equal to $x$, and for each element in $(\mathbf{Z} / q \mathbf{Z})^{*}$, we take the representative $1 \leq a \leq q-1$.

Remark 3.1. By Xue and Zarhin [9, Proposition 2.1, 2.2], the assumptions (A)-(C) of Theorem 1.1 guarantee that there exists an integer $a$ such that

$$
1 \leq a \leq q-1, \quad \operatorname{gcd}(a, p)=1
$$

and the integers $[n a / q]$ and $\operatorname{dim}_{E} V=n-1$ are relative prime. We note that the conditions $(\mathrm{A})-(\mathrm{C})$ of Theorem 1.1 are equivalent to the conditions $(\mathrm{A})-(\mathrm{C})$ of $[9$, Theorem 0.1]. 
Since $V=H_{1}(X, \mathbf{Q})$ carries a natural structure of $E$-vector space, the first complex homology group $V_{\mathbf{C}}=H_{1}(X, \mathbf{C})=H_{1}(X, \mathbf{Q}) \otimes_{\mathbf{Q}} \mathbf{C}$ carries a structure of $E_{\mathbf{C}}$-module, and therefore splits into a direct sum

$$
V_{\mathbf{C}}=\bigoplus_{a \in G} V_{a}
$$

Each $V_{a}$ is a $\mathbf{C}$-vector space of dimension $\operatorname{dim}_{E} V=n-1$.

There is a canonical Hodge decomposition [1, pp. 52-53; 3, Chapter 1]

$$
V_{\mathbf{C}}=H_{1}(X, \mathbf{C})=H^{-1,0}(X) \oplus H^{0,-1}(X),
$$

where $H^{-1,0}(X)$ and $H^{0,-1}(X)$ are $\operatorname{dim}(X)$-dimensional complex vector spaces that are mutually "complex conjugate". This splitting is $E$-invariant, and $H^{-1,0}(X)$ and $\operatorname{Lie}(X)$ are canonically isomorphic as $E_{\mathbf{C}}$-modules. In particular,

$$
\operatorname{dim}_{\mathbf{C}} H^{-1,0}(X)_{a}=\operatorname{dim}_{\mathbf{C}} \operatorname{Lie}(X)_{a}=n_{a} .
$$

Let $\mathfrak{f}_{H}^{0}=\mathfrak{f}_{H, Z}^{0}: V_{\mathbf{C}} \rightarrow V_{\mathbf{C}}$ be the $\mathbf{C}$-linear operator such that

$$
\mathfrak{f}_{H}(x)=-x / 2 \quad \forall x \in H^{-1,0}(X) ; \quad \mathfrak{f}_{H}^{0}(x)=x / 2, \quad \forall x \in H^{0,-1}(X) .
$$

Since the Hodge decomposition is $E$-invariant, $\mathfrak{f}_{H}^{0}$ commutes with $E$. Therefore, each $V_{a}$ is $\mathfrak{f}_{H}^{0}$-invariant. It follows that the linear operator $\mathfrak{f}_{H}^{0}: V_{a} \rightarrow V_{a}$ is semisimple and its spectrum lies in the two-element set $\{-1 / 2,1 / 2\}$. The multiplicity of eigenvalue $-1 / 2$ is $n_{a}=\operatorname{dim}_{\mathbf{C}} H^{-1,0}(X)_{a}$, while the multiplicity of eigenvalue $1 / 2$ is $\operatorname{dim}_{E} V-n_{a}$. Clearly, the complex conjugate of $a \in \operatorname{Gal}(E / \mathbf{Q})=(\mathbf{Z} / q \mathbf{Z})^{*}$ is $\bar{a}=q-a$. It is known $[1,2]$ that

$$
n_{a}+n_{\bar{a}}=\operatorname{dim}_{E} V .
$$

This implies that the multiplicity of the eigenvalue $1 / 2$ coincides with $n_{\bar{a}}$.

The Hodge-Lie algebra hdg of $X$ is a reductive $\mathbf{Q}$-Lie subalgebra of $\operatorname{End}_{\mathbf{Q}}(V)$. Its natural representation in $V$ is completely reducible and its centralizer in $\operatorname{End}_{\mathbf{Q}}(V)$ coincides with $\operatorname{End}^{0}(X)=E$. Moreover, its complexification

$$
\operatorname{hdg}_{\mathbf{C}}=\operatorname{hdg} \otimes_{\mathbf{Q}} \mathbf{C} \subset \operatorname{End}_{\mathbf{Q}}(V) \otimes_{\mathbf{Q}} \mathbf{C}=\operatorname{End}_{\mathbf{C}}\left(V_{\mathbf{C}}\right)
$$

contains $\mathfrak{f}_{H}^{0}\left[8\right.$, Section 3.4]. Recall that hdg $=\mathfrak{c} \oplus \mathrm{hdg}^{\mathrm{ss}}$, with $\mathfrak{c}$ being the center of hdg and $\mathrm{hdg}^{\mathrm{ss}}=\left[\mathrm{hdg}\right.$, hdg] the semisimple part. Let $\mathfrak{c}_{\mathbf{C}}:=\mathfrak{c} \otimes_{\mathbf{Q}} \mathbf{C}$ be the complexification of $\mathfrak{c}$ and $\operatorname{hdg}_{\mathbf{C}}^{\mathrm{ss}}:=\mathrm{hdg}^{\mathrm{ss}} \otimes_{\mathbf{Q}} \mathbf{C}$ the complexification of $\mathrm{hdg}^{\mathrm{ss}}$. Clearly, $\operatorname{hdg}^{\mathrm{ss}} \subset \mathfrak{s l}_{E}(V)$, and thus

$$
\operatorname{hdg}_{\mathbf{C}}^{\mathrm{ss}} \subset \mathfrak{s l}_{E_{\mathbf{C}}}\left(V_{\mathbf{C}}\right)=\bigoplus_{a \in G} \mathfrak{s l}_{\mathbf{C}}\left(V_{a}\right)
$$

We write $\operatorname{hdg}_{a}^{\mathrm{ss}}$ for the image of projection $P_{a}: \operatorname{hdg}_{\mathbf{C}}^{\mathrm{ss}} \rightarrow \mathfrak{s l}_{\mathbf{C}}\left(V_{a}\right)$. Each $\operatorname{hdg}_{a}^{\mathrm{ss}}$ is a semisimple complex Lie subalgebra of $\mathfrak{s l}_{\mathbf{C}}\left(V_{a}\right)$.

Remark 3.2. Let us decompose $f_{H}^{0}$ as $f+f^{\prime}$ with $f^{\prime} \in \mathfrak{c}_{\mathbf{C}}$ and $f \in \operatorname{hdg} \mathrm{g}_{\mathbf{C}}^{\text {ss }}$. By Xue and Zarhin [9, Remark 3.2], the natural representation $V_{a}$ of $\operatorname{hdg}_{a}^{\mathrm{ss}}$ is simple for all $a \in G$. It follows from Schur's Lemma that when restricted to each $V_{a}, f^{\prime}$ coincides with multiplication by scalar $c_{a} \in \mathbf{C}$. Therefore, $\operatorname{hdg}_{\mathbf{C}}^{\text {ss }}$ contains an operator (namely, $f$ ) whose restriction on each $V_{a}$ is diagonalizable with at most two eigenvalues: $-1 / 2-c_{a}$ of multiplicity $n_{a}$ and $1 / 2-c_{a}$ of multiplicity $n_{\bar{a}}=\operatorname{dim}_{E} V-n_{a}$. 
Lemma 3.1. Let the assumptions be the same as in Theorem 1.1. There exists an $a \in G=(\mathbf{Z} / q \mathbf{Z})^{*}$ such that $\operatorname{hdg}_{a}^{\mathrm{ss}}=P_{a}\left(\operatorname{hdg}_{\mathbf{C}}^{\mathrm{ss}}\right)$ coincides with $\mathfrak{s l}_{\mathbf{C}}\left(V_{a}\right)$.

Proof. The idea is to combine Remarks 3.1, 3.2 together with Lemma 3.3 of [9]. This result is already contained in the proof of [9, Theorem 3.4], where we note that the assumption $n>q$ in [9, Theorem 3.4] is not used for this particular step of the proof.

Notice that this is the place where assumptions $(\mathrm{A})-(\mathrm{C})$ in Theorem 1.1 are used, since we need to make sure that there exists $a \in G$ such that $n_{a}$ and $\operatorname{dim}_{E} V$ are relative prime in order to apply Lemma 3.3 of [9].

Let $h:(\mathbf{Z} / q \mathbf{Z})^{*} \rightarrow \mathbf{R}$ be the function such that for all $1 \leq a \leq q-1$ with $\operatorname{gcd}(a, q)=1$

$$
h(a)=\left(\frac{\operatorname{dim}_{E} V}{2}-n_{a}\right)^{2}=\left(\frac{n-1}{2}-\left[\frac{n a}{q}\right]\right)^{2} .
$$

By (3.1), $n_{a}+n_{\bar{a}}=\operatorname{dim}_{E} V$, so $h(a)=h(\bar{a})=h(q-a)$, which is also easy to check directly from (3.2). The function $h$ is nonincreasing on the set of integers

$$
[1, q / 2]_{\mathbf{Z}}:=\{a \mid 1 \leq a \leq q / 2, \operatorname{gcd}(a, p)=1\} .
$$

By Remark 1.1, we have $4 \leq n<q$. In particular, $[n / q]=0$. On the other hand, let $t$ be the maximal element of $[1, q / 2]_{\mathbf{Z}}$. Then $t \neq 1$ and $[n t / q] \neq 0$. It follows that $h$ is not a constant function.

Lemma 3.2. Let the assumption be the same as Theorem 1.1. Let $(a, b) \in G^{2}$ be $a$ pair such that $h(a) \neq h(b)$. Then $P_{a, b}\left(\operatorname{hdg}_{\mathbf{C}}^{\mathrm{ss}}\right)=\mathfrak{s l}_{\mathbf{C}}\left(V_{a}\right) \oplus \mathfrak{s l}_{\mathbf{C}}\left(V_{b}\right)$.

Proof. By (3.2),

$$
h(a)-h(b)=\left(n_{a}-n_{b}\right)\left(\operatorname{dim}_{E} V-n_{a}-n_{b}\right) .
$$

So $h(a) \neq h(b)$ if and only if $n_{a} \neq n_{b}$ and $n_{a} \neq \operatorname{dim}_{E} V-n_{b}$. Let $\mathfrak{k}^{\text {ss }}=P_{a, b}\left(\operatorname{hdg}_{\mathbf{C}}^{\text {ss }}\right)$. By Lemma 3.1 and part (i) of Lemma 2.1, both projections $\mathfrak{k}^{\text {ss }} \rightarrow \mathfrak{s l}_{\mathbf{C}}\left(V_{a}\right)$ and $\mathfrak{k}^{\text {ss }} \rightarrow$ $\mathfrak{s l}_{\mathbf{C}}\left(V_{b}\right)$ are surjective. By Remark 3.2, $P_{a, b}(f)$ is a semisimple element of $\mathfrak{k}^{s s} \subseteq$ $\operatorname{End}_{\mathbf{C}}\left(V_{a}\right) \oplus \operatorname{End}_{\mathbf{C}}\left(V_{b}\right)$ such that $P_{a, b}(f)$ acts on $V_{a}$ with (at most) two eigenvalues of multiplicities $n_{a}$ and $\operatorname{dim}_{E} V-n_{a}$, respectively, and similarly for $b$. Lemma 3.2 follows by setting $d=2$ in [9, Lemma 3.6]. Last, we point out that the assumption that the multiplicities $a_{i}$ are positive in [9, Lemma 3.6] is not used in its proof, so the lemma applies even if $n_{a}$ or $n_{b}$ is zero, which may happen if $n<q$.

Proof of Theorem 1.1. As remarked at the end of Section 2, Theorem 1.1 follows if we show that conditions (I) and (II) of Lemma 2.2 holds for $\mathfrak{k}=\mathrm{hdg}^{\mathrm{ss}}$. Condition (I) holds by Lemma 3.1. To show that condition (II) holds, by Lemma 3.2 it is enough to prove that for each $(a, b) \in G^{2}$ with $a \neq b$ and $a \neq \bar{b}$, there exists $x \in G$ such that $h(x a) \neq h(x b)$. Suppose that this is not the case, then there exists a pair $(a, b)$ such that $h(x a)=h(x b)$ for all $x \in G$. Without loss of generality, we may and will assume that $b=1 \in(\mathbf{Z} / q \mathbf{Z})^{*}$, thus $a \neq \pm 1$. It follows that $h(x a)=h(x)$ for all $x \in(\mathbf{Z} / q \mathbf{Z})^{*}$. Since $h$ is not a constant function, such an $a$ does not exists by Lemma 4.1 of next section. Contradiction. 


\section{Arithmetic results}

Throughout this section, $G=(\mathbf{Z} / q \mathbf{Z})^{*}$. For each $a \in G$, let $\theta_{a}: G \rightarrow G$ be the translation map: $b \mapsto a b$. A real valued function $h: G \rightarrow \mathbf{R}$ is said to be even if $h \circ \theta_{-1}=h$. For any $x \leq y \in \mathbf{R}$, we write $[x, y]_{\mathbf{z}}$ for the set of integers $\{i \mid x \leq i \leq$ $y, \operatorname{gcd}(i, p)=1\}$.

Lemma 4.1. Let $h:(\mathbf{Z} / q \mathbf{Z})^{*} \rightarrow \mathbf{R}$ be an even function that's monotonic on $[1, q / 2]_{\mathbf{Z}}$. If $h \circ \theta_{a}=h$ for some $a \in(\mathbf{Z} / q \mathbf{Z})^{*}$ and $a \neq \pm 1$, then $h$ is a constant function.

Proof. We prove the lemma in seven steps.

Step 1. Let $\langle \pm a\rangle$ be the subgroup of $(\mathbf{Z} / q \mathbf{Z})^{*}$ generated by $a$ and -1 . Clearly, $h \circ \theta_{b}=h$ for any $b \in\langle \pm a\rangle$ since $h \circ \theta_{a}=h$ and $h$ is even. In particular, this holds true for the maximal element $b_{\max }$ in the set in $\langle \pm a\rangle \cap[1, q / 2]_{\mathbf{z}}$. If $b_{\max }=1$, the group $\langle \pm a\rangle$ is necessarily $\{ \pm 1\}$. Therefore, it is enough to prove that $h$ being nonconstant implies that $b_{\max }=1$. So without loss of generality, we assume that $a=b_{\max }$ throughout the rest of the proof. Notice that if $a \neq 1$, then $2 a^{2}>q$, otherwise it contradicts the maximality of $a$.

Step 2. Lemma 4.1 holds if $p=2$.

Every even function on $(\mathbf{Z} / q \mathbf{Z})^{*}$ is constant if $q$ is 2 or 4 so we assume that $q=$ $2^{r} \geq 8$. The group $\left(\mathbf{Z} / 2^{r} \mathbf{Z}\right)^{*}$ is isomorphic to $\mathbf{Z} / 2 \mathbf{Z} \times \mathbf{Z} / 2^{r-2} \mathbf{Z}$, where the factor $\mathbf{Z} / 2 \mathbf{Z}$ is generated by -1 . Let us assume that $\langle \pm a\rangle$ has order $2^{s}$. Since $\langle \pm a\rangle \supseteq\langle \pm 1\rangle$, it follows that $\langle \pm a\rangle \cong \mathbf{Z} / 2 \mathbf{Z} \times \mathbf{Z} / 2^{s-1} \mathbf{Z}$. In particular, if $\langle \pm a\rangle \neq\langle \pm 1\rangle$, then $\mathbf{Z} / 2^{s-1} \mathbf{Z}$ is nontrivial, therefore $\langle \pm a\rangle$ contains three elements of order two. But there are exactly 3 elements of order two in $(\mathbf{Z} / q \mathbf{Z})^{*}:-1,2^{r-1}-1,2^{r-1}+1$. Hence $\langle \pm a\rangle$ contains all the above elements of order 2 . So $a=2^{r-1}-1$ since it is the largest element in $[1, q / 2]_{\mathbf{z}}$. Therefore,

$$
h(q / 2-1)=h\left(2^{r-1}-1\right)=h(a)=\left(h \circ \theta_{a}\right)(1)=h(1) .
$$

Since $h$ is monotonic on $[1, q / 2]_{\mathbf{z}}$, the above equality implies that $h$ is constant on $[1, q / 2]_{\mathbf{Z}}$ and therefore a constant function.

Step 3. Let $p$ be an odd prime. Lemma 4.1 holds if either $a$ is even, or $a$ is odd and $3 a \geq q$.

It is enough to prove that if $a \neq 1$, then $h(1)=h((q-1) / 2)$. Since $h(1)=$ $\left(h \circ \theta_{a}\right)(1)=h(a)$, by monotonicity $h$ is constant on $[1, a]_{\mathbf{Z}}$. Therefore, it is enough to find $b$ such that $h((q-1) / 2)=h(b)$ and $b \in[1, a]_{\mathbf{z}}$.

First, let us assume that $a=2 b$ is even. Then

$$
a \cdot \frac{q-1}{2}=(q-1) b \equiv-b \bmod q .
$$

So, $h((q-1) / 2)=h(a(q-1) / 2)=h(-b)=h(b)$. Clearly $b=a / 2$ lies in $[1, a]_{\mathbf{z}}$.

Next, assume that $a$ is odd. Then

$$
a \cdot \frac{q-1}{2}=\frac{q a-a}{2} \equiv \frac{q-a}{2} \quad(\bmod q) .
$$

So, $h((q-1) / 2)=h((q-a) / 2)$. Let $b=(q-a) / 2$. When $3 a \geq q$, we have $b=$ $(q-a) / 2 \leq a$ hence $b$ lies in $[1, a]_{\mathbf{z}}$ as desired. 
Step 4. Lemma 4.1 holds if $p=3$.

When $p$ is odd, $\left(\mathbf{Z} / p^{r} \mathbf{Z}\right)^{*}$ is cyclic of order $\varphi\left(p^{r}\right)=(p-1) p^{r-1}$. For $p=3$,

$$
\left(\mathbf{Z} / 3^{r} \mathbf{Z}\right)^{*} \cong \mathbf{Z} /\left(2 \cdot 3^{r-1}\right) \mathbf{Z} \cong \mathbf{Z} / 2 \mathbf{Z} \times \mathbf{Z} / 3^{r-1} \mathbf{Z}
$$

In particular, if $q \geq 9,(\mathbf{Z} / q \mathbf{Z})^{*}$ contains a unique subgroup of order 3 which is generated by $3^{r-1}+1$. If the order of $\langle \pm a\rangle$ is coprime to 3 , then $\langle \pm a\rangle$ is necessarily $\{ \pm 1\}$, which leads to a contradiction to the assumption of the lemma. If the order of $\langle \pm a\rangle$ is divisible by 3 , then $q \geq 9$ and $\langle \pm a\rangle$ contains $3^{r-1}+1$. By assumption on the maximality of $a$ we must have $a \geq 3^{r-1}+1$ and hence $3 a>q$.

Step 5. Assume that both $p$ and $a$ are odd, $p \neq 3$ and $3 a<q$. Lemma 4.1 holds if $7 a \geq q$.

Since $p \neq 3,(q-3) / 2$ lies in $[1, q / 2]_{\mathbf{z}}$. It is enough to prove that $a \neq 1$ implies that $h(1)=h((q-3) / 2)$. Indeed, it follows from the proof of Step 3 that $h((q-1) / 2)=$ $h((q-a) / 2)$. But if $a \neq 1$ then $a \geq 3$ so $(q-a) / 2 \leq(q-3) / 2$. If we prove that $h$ is constant on $[1,(q-3) / 2]_{\mathbf{z}}$, then $h((q-1) / 2)=h((q-a) / 2)=h(1)$ and it follows that $h$ is a constant function.

By our assumption $3 a<q$, so $(q-3 a) / 2$ lies in $[1, q / 2] \mathbf{z}$. Notice that

$$
a \cdot \frac{q-3}{2} \equiv \frac{q-3 a}{2} \bmod q \text {. }
$$

We see that $h((q-3) / 2)=h((q-3 a) / 2)$. If $a \geq(q-3 a) / 2$, then $h(1)=h((q-3 a) / 2)$ since $h$ is constant on $[1, a]_{\mathbf{Z}}$, or else $a<(q-3 a) / 2$ so $5 a<q$. In this case, $2 a<q / 2$. But $2 \in[1, a]_{\mathbf{Z}}$ since $p$ is odd and $a \geq 3$. So $h(2)=h(1)$, therefore $h(2 a)=h(1)$ and $h$ is constant on $[1,2 a]_{\mathbf{z}}$. But now by our assumption $7 a \geq q$, or equivalently $2 a \geq(q-3 a) / 2$, it follows that:

$$
h\left(\frac{q-3}{2}\right)=h\left(\frac{q-3 a}{2}\right)=h(1) .
$$

Step 6. Assume that both $p$ and $a$ are odd, $p \neq 3,5$ and $7 a<q$. Lemma 4.1 holds.

Since $7 a<q$ and $p \neq 5,(q-5 a) / 2$ lies in $[1, q / 2] \mathbf{z}$. By similar argument as in Step $5, h((q-5) / 2)=h((q-5 a) / 2)$. We claim that now it is enough to show that $h(1)=h((q-5) / 2)$. Indeed, by the proof of the Step 5 , all we need to show is that $h(1)=h((q-3) / 2)$, but since $a \geq 3$, then $(q-3 a) / 2<(q-5) / 2$. So, $h$ being constant on $[1,(q-5) / 2]_{\mathbf{z}}$ implies that $h(1)=h((q-3 a) / 2)=h((q-3) / 2)$.

Let $S$ be the set of integers

$$
S=\{b \mid b \geq 1, p \nmid b,(2 b+1) a<q\} .
$$

Clearly $1 \in S$ so $S$ is not empty. Let $x$ be the maximal element of $S$. By Step 1 , $2 a^{2}>q$ so necessarily $x<a$. Since $h$ is constant on $[1, a]_{\mathbf{z}}$, we must have $h(1)=h(x)$. Notice that $x a<q / 2$ by assumption. So, $h(a x)=h(x)=h(1)$ and it follows that $h$ is constant on $[1, a x]_{\mathbf{z}}$. Assume that $h(1) \neq h((q-5) / 2)$. It is necessary that $a x<(q-5 a) / 2$, or equivalently, $(2 x+5) a<q$. But we can choose $x^{\prime}$ from the two elements set $\{x+1, x+2\}$ such that $x^{\prime}$ is coprime to $p$. It follows that $x^{\prime} \in S$. This contradicts the maximality of $x$.

Step 7. Lemma 4.1 holds if $p=5$. 
If the order of $\langle \pm a\rangle$ is divisible by 5 , then $\langle \pm a\rangle$ contains the unique subgroup of order 5 in $\left(\mathbf{Z} / 5^{r} \mathbf{Z}\right)^{*}$. In particular, $2 \times 5^{r-1}+1 \in\langle \pm a\rangle$. It follows that $a>2 \times 5^{r-1}+1$ and therefore $3 a>5^{r}$. The Lemma holds by Step 3 .

If the order of $\langle \pm a\rangle$ is coprime to 5 . Then from the isomorphism

$$
\mathbf{Z} / 5^{r} \mathbf{Z} \cong \mathbf{Z} / 4 \mathbf{Z} \times \mathbf{Z} / 5^{r-1} \mathbf{Z},
$$

we see that $\langle \pm a\rangle$ is has either order 2 or 4 . If $\langle \pm a\rangle$ has order 2 , then $\langle \pm a\rangle$ is necessarily $\langle \pm 1\rangle$ and this leads to a contradiction. So, we assume that $\langle \pm a\rangle$ has order 4 and $a$ is the unique element such that $1<a<5^{r} / 2$ and $a^{2} \equiv-1 \bmod 5^{r}$. In particular, $a^{2}+1 \geq 5^{r}$. If $a$ is even then the Lemma holds by Step 3. In particular, this works for $q=p=5$ since $a=2$ in this case. We assume that $q \geq 25$ and $a$ is odd through out the rest of the proof. First, we claim that $a \geq 7$. Indeed, if $q=25$, then $a=7$ by direct calculation; if $q>25$, then $a>7$ since $a^{2}+1 \geq q$. This implies that $(q-a) / 2 \leq(q-7) / 2$. Therefore, it is enough to prove that $h((q-7) / 2)=h(1)$ since it then follows that $h((q-1) / 2)=h((q-a) / 2)=h(1)$. By Step 5 we may also assume that $7 a<q$. It follows that $(q-7 a) / 2 \in[1, q / 2]_{\mathbf{Z}}$ and $h((q-7) / 2)=h((q-7 a) / 2)$.

Let $c=[q / a]$. Since $a^{2}+1 \geq q$ and $a<q / 2$, we see that $2 \leq c \leq a$. Let $x=[c / 2]$ if $[c / 2]$ is not divisible by 5 , and $x=[c / 2]-1$ otherwise. Notice that $a>x \geq \max \{1,(c-3) / 2\}$ and $x a \leq q / 2$ by our choice of $x$. It follows that $x \in[1, a]_{\mathbf{z}}$, therefore $h(x)=h(1)$, and therefore $h(a x)=h(x)=h(1)$. So $h$ is constant on $[1, a x]_{\mathbf{Z}}$. If $h(1) \neq h((q-7) / 2)$, we must have $x a<(q-7 a) / 2$, or equivalently, $(2 x+7) a<q$. Then it follows that:

$$
\frac{q}{a}>2 x+7 \geq 2\left(\frac{c-3}{2}\right)+7=c+4=\left[\frac{q}{a}\right]+4
$$

which is absurd.

Lemma 4.1 is proved by combining all the above steps.

\section{Acknowledgments}

The author is supported by an NCTS postdoctoral fellowship. He would also like to express his gratitude to Yuri G. Zarhin, who has provided valuable suggestions.

\section{References}

[1] P. Deligne, Hodge cycles on abelian varieties (notes by J. S. Milne), Lect. Notes Math. 900, Springer-Verlag, Berlin, Heidelberg, New York, 1982, 9-100.

[2] B. Moonen and Yu.G. Zarhin, Weil classes on abelian varieties, J. Reine Angew. Math. 496 (1998), 83-92.

[3] D. Mumford, Abelian varieties, 2nd ed. Oxford University Press, London, 1974.

[4] K. Ribet, Galois action on division points of Abelian varieties with real multiplications. Amer. J. Math. 98 (1976), 751-804.

[5] K. Ribet, Hodge classes on certain abelian varieties, Amer. J. Math. 105 (1983), 523-538.

[6] D. Ulmer and Y. G. Zarhin, Rank of Jacobians in towers of function fields, Math. Res. Lett. 17(4) (2010), 637-645.

[7] J. Xue, Endomorphism algebras of Jacobians of certain superelliptic curves, J. Number Theory 131 (2011), 332-342.

[8] J. Xue and Y. G. Zarhin, Centers of Hodge groups of superelliptic Jacobians, Transform. Groups 15(2) (2010), 449-482.

[9] J. Xue and Y. G. Zarhin, Hodge groups of certain superelliptic Jacobians, Math. Res. Lett. $\mathbf{1 7}(2)$ (2010), 371-388. 
[10] Y. G. Zarhin, Weights of simple Lie algebras in the cohomology of algebraic varieties, Izv. Akad. Nauk SSSR Ser. Mat. 48 (1984), 264-304; Math. USSR Izv. 24 (1985), 245-281.

[11] Y. G. Zarhin, Hyperelliptic jacobians without complex multiplication, Math. Res. Lett. 7 (2000), $123-132$.

[12] Y. G. Zarhin, Very simple 2-adic representations and hyperelliptic jacobians, Moscow Math. J. 2(2) (2002), 403-431.

[13] Y. G. Zarhin, Cyclic covers, their Jacobians and endomorphisms, J. Reine Angew. Math. 544 (2002), 91-110.

[14] Y. G. Zarhin, The endomorphism rings of Jacobians of cyclic covers of the projective line, Math. Proc. Cambridge Philos. Soc. 136 (2004), 257-267.

[15] Y. G. Zarhin, Endomorphism algebras of superelliptic Jacobians, in 'Geometric methods in algebra and number theory', eds. F. Bogomolov and Y. Tschinkel, Progress in Math. 235, Birkhäuser, Boston Basel, Berlin, 2005, 339-362.

[16] Y. G. Zarhin, Superelliptic jacobians, in 'Diophantine geometry' (ed. U. Zannier), Proceedings, Edizioni Della Normali, Pisa, 2007, 363-390.

[17] Y. G. Zarhin, Endomorphisms of superelliptic jacobians, Math. Z. 261 (2009), 691-707, 709.

National Center for Theoretical Sciences, Mathematical Division, National Tsing Hua University, Third General Building, No.101, Sec 2, Kuang Fu Road, Hsinchu, Taiwan 30043, TAIWAN R.O.C.

E-mail address: xue_j@math.cts.nthu.edu.tw 